\title{
Disseminating clot burden post- craniotomy: The difficult balancing act of clot versus haemorrhage post-neurosurgery
}

\author{
Emma Bowcock, Idunn Morris ${ }^{1}$, Stuart Lane ${ }^{2}$
}

\begin{abstract}
Venous thromboembolism (VTE) is the most common complication following craniotomy for neoplastic disease. Its occurrence is associated with a significant morbidity and mortality, and balancing the risks for the subsequent development ofVTE versus intracranial haemorrhage $(\mathrm{ICH})$ can lead to difficult management decisions for treating clinicians. We present a case ofVTE following craniotomy for meningioma complicated by ICH in the presence of a disseminating clot burden that included pulmonary, intra-cardiac and paradoxical arterial embolic sequelae. Management strategies incorporated pharmacological, radiological and surgical methods. We discuss the evidence forVTE prevention and treatment, as well as the role of inferior vena cava filters and thrombectomy. We finally highlight the use of desmopressin as a potential risk factor for VTE, and encourage the need for an individualised approach to peri-operative risk stratification in the neurosurgical intensive care population.
\end{abstract}

Key words: Anticoagulation, inferior vena cava filter, neurosurgical intensive care, thrombectomy, venous thromboembolism

\section{CASE REPORT}

A 55-year-old female presented to the emergency department with a 1-month history of headache and short-term memory loss. Neurological examination was unremarkable. An initial computed tomography (CT) brain and subsequent magnetic resonance imaging brain demonstrated a left occipito-parietal mass, $6 \mathrm{~cm}$ in diameter with associated $8 \mathrm{~mm}$ midline shift [Figure 1]. Relevant past medical history included hypertension and surgical excision of a melanoma. On day 3 after Intensive Care Registrar, Nepean Hospital, ${ }^{2}$ Staff Specialist in Intensive Care, Sydney Medical School, Nepean Hospital, ${ }^{1}$ ICU Registrar, Nepean Hospital, Sydney, NSW, Australia

Address for correspondence:

Dr. Emma Bowcock, 802/242 Elizabeth Street, Surry Hills,

Sydney, NSW 2010, Australia.

E-mail: emma.bowcock@hotmail.com

\begin{tabular}{|l|l|}
\hline \multicolumn{2}{|c|}{ Access this article online } \\
\hline Quick Response Code: & Website: \\
\hline & www.jnaccjournal.org \\
\hline & \\
\hline
\end{tabular}

initial embolisation, she underwent stereotactic craniotomy and resection of the meningioma. CT brain on day 4 showed no residual tumour and no post-operative bleeding. She was commenced on subcutaneous unfractionated heparin (UFH) 5000 units twice daily. Sequential calf compression devices had been applied on day 2. Her immediate post-operative course was complicated by the development of diabetes insipidus, which was treated with two intravenous boluses of $0.25 \mathrm{mcg}$ desmopressin. She was discharged home on day 12, and heparin prophylaxis was stopped upon discharge.

On day 16, she presented to her general practitioner with increasing breathlessness. An outpatient CT pulmonary angiogram (CTPA) performed on the same

This is an open access article distributed under the terms of the Creative Commons Attribution-NonCommercial-ShareAlike 3.0 License, which allows others to remix, tweak, and build upon the work non-commercially, as long as the author is credited and the new creations are licensed under the identical terms.

For reprints contact: reprints@medknow.com

How to cite this article: Bowcock E, Morris I, Lane S. Disseminating clot burden post- craniotomy: The difficult balancing act of clot versus haemorrhage post-neurosurgery. J Neuroanaesthesiol Crit Care 2016;3:252-8. 
day demonstrated bilateral pulmonary embolism (PE), and she was transferred directly to the emergency department. On examination, her vital signs were heart rate of 97 beats/ $\mathrm{min}$, blood pressure of $120 / 80 \mathrm{mmHg}$, respiratory rate of 20 breaths / $\mathrm{min}$ and oxygen saturation of $92 \%$ on room air. She was commenced on $80 \mathrm{mg}$ enoxaparin subcutaneously twice daily based on a weight of $79 \mathrm{~kg}$. CT brain on day 17 demonstrated a $6 \mathrm{~mm}$ mixed density subdural haematoma (SDH) with a hypodense component posterosuperiorly [Figure 2], with no change in her clinical condition. She was transferred to the Intensive Care Unit (ICU), and her anticoagulation regimen was changed to an UFH infusion titrated to an activated partial thromboplastin time (APTT) of 60-80 s. Intravenous UFH was used in preference to low-molecular weight heparins (LMWHs) because of the potential ability to rapidly reverse anti-coagulation in the event of SDH extension. A narrower therapeutic APTT range (usually 60-100 s in this institution) was chosen to minimise the risk of further haemorrhage. Lower limb venous Doppler on day 18 showed a non-occlusive $3 \mathrm{~cm}$ deep vein thrombosis (DVT) of the right mid-femoral vein. A transthoracic echocardiogram (TTE) showed no evidence of the right ventricular (RV) strain with normal pulmonary artery pressures. She was discharged from the ICU on day 20.

Maintaining optimal anticoagulation in the ICU and subsequently on the ward was difficult [Figure 3], with frequent sub-therapeutic APTT levels, the lowest being 32 s. On day 24, repeat lower limb venous Doppler showed proximal and distal extension of the right femoral DVT, and a repeat CT brain showed the extension of the SDH (increase in diameter from 6 to $13 \mathrm{~mm}$, with increased midline shift from 6 to $9 \mathrm{~mm}$ ). Later that day, she developed tachypnoea and hypoxia while mobilising, in association with an acutely painful and swollen left leg. She was re-admitted to the ICU, and a CT angiogram of the lower limbs demonstrated extensive clot at the aortic bifurcation, with extension into and complete occlusion of the left common iliac artery and 50\% occlusion of the right common femoral artery [Figure 4]. TTE with bubble contrast demonstrated a patent foramen ovale (PFO), moderately severe pulmonary hypertension, moderate RV enlargement and normal RV systolic motion, suggesting further PE. Her vital signs at the time were blood pressure of $149 / 59 \mathrm{mmHg}$, heart rate of 91 beats/min, respiratory rate of 25 breaths/min and oxygen saturation of $91 \%$ on $\mathrm{FiO}_{2}$ of 0.5 administered via high-flow nasal prongs. She was transferred to the operating theatre for aorto-iliac thrombectomy and placement of an inferior vena cava (IVC) filter. She was continued on a UFH infusion alongside the IVC filter, as the risk of further thromboembolic events was deemed extremely high. Repeat CT brain on day 25 showed no change in the size of the SDH, and she

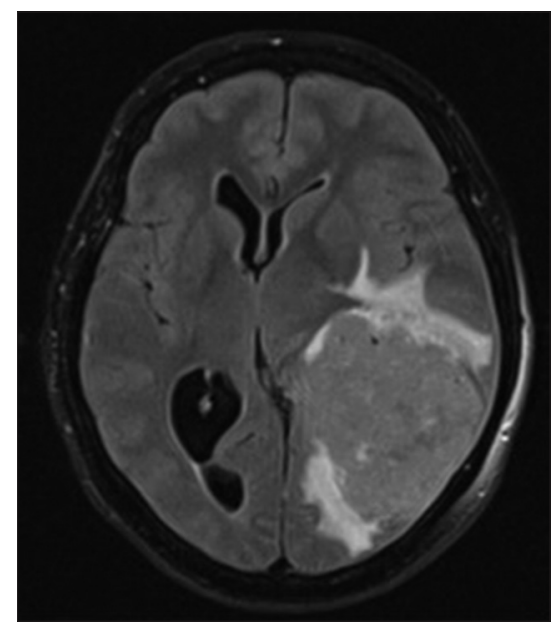

Figure 1: Transverse slice of pre-operative magnetic resonance imaging T2 flair, demonstrating large occipito-parietal meningioma

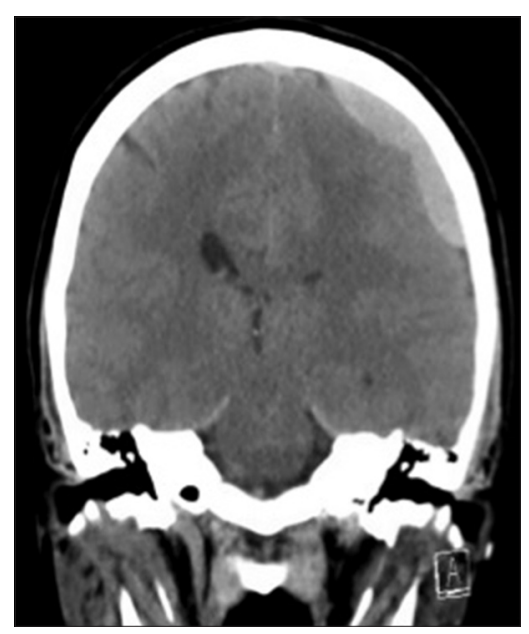

Figure 2: Coronal slice of computerised tomography brain showing subdural haematoma following the commencement of therapeutic low molecular weight heparin

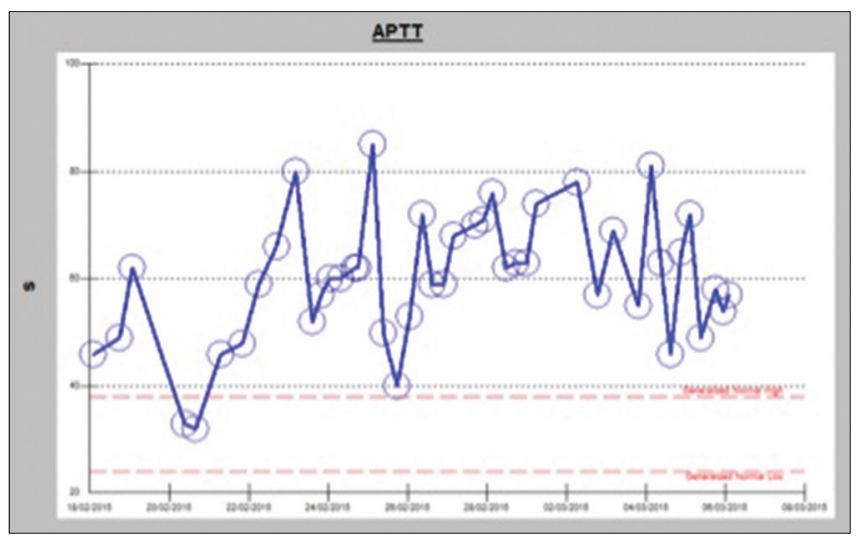

Figure 3: Activated partial thromboplastin time results during the initial period of anticoagulation

was discharged back to the ward on day 32. On day 35, she had an episode of decreased level of consciousness and worsening hypoxia. CTPA showed extensive 
bilateral pulmonary emboli, with a large thrombus in the right atrium extending through the PFO into the left atrium [Figure 5]. TTE showed $45 \mathrm{~mm} \times 13 \mathrm{~mm}$ thrombus in the right atrium, severe RV dilatation, severe RV systolic dysfunction and estimated pulmonary artery systolic pressures of $70 \mathrm{mmHg}$ [Figure 6]. She was transferred to a quaternary cardiothoracic centre and underwent bi-atrial thrombectomies, closure of PFO and left pulmonary endarterectomy. She was transitioned back from intravenous UFH to therapeutic dose LMWH (enoxaparin $80 \mathrm{mg}$ BD) on day 48 and discharged home on day 52 on therapeutic enoxaparin, which she continued for a total duration of 9 months.

Despite being found to be heterozygous for factor $\mathrm{V}$ Leiden, the haematologist felt that this was a provoked event and lifelong anticoagulation was not required. Her most recent outpatient TTE showed normal RV function with no evidence of pulmonary arterial hypertension. She made a good neurological and functional recovery, and she is currently awaiting removal of the IVC filter.

\section{DISCUSSION}

Patients who undergo craniotomy due to central nervous system malignancy have a high incidence of developing venous thromboembolism (VTE) complications ${ }^{\left[{ }^{[1]}\right.}$ In a 10-year retrospective cohort study of 1148 patients, the rates of DVT and PE were $13.7 \%$ and $3.3 \%$, respectively. ${ }^{[2]} \mathrm{PE}$ is often a fatal complication with a reported mortality ranging from $9 \%$ to $50 \% .{ }^{[3]}$ Risk factors for VTE in patients undergoing craniotomy include the presence of malignancy, large tumour size, duration of surgery $>4 \mathrm{~h}$, lower limb paresis, increased age and use of chemotherapy. ${ }^{[4]}$ The majority of thromboembolic complications occur within the first post-operative week, which supports the use of early prophylactic therapy ${ }^{[5]}$

\section{Venous thromboembolism prophylaxis and treatment}

Mechanical prophylaxis of VTE includes the use of intermittent pneumatic compression devices (IPC) and elasticated stockings (ES). These devices are thought to work by reducing the overall cross-sectional area of the limb, increasing the linear velocity of venous flow, reducing venous wall distension and improving valvular function. ${ }^{[6]}$ The antithrombotic effect of IPC is suggested to be the result of stimulation of endogenous fibrinolysis. ${ }^{[7]}$ Skillman et al. compared IPC to no mechanical prophylaxis in neurosurgical patients and showed significantly lower rates of DVT in those treated with IPC. ${ }^{[8]}$ A systematic review of IPC versus ES by Morris and Woodcock suggested a benefit of IPC over $\mathrm{ES}^{\left[{ }^{[9]}\right.}$ however Turpie et al. demonstrated that there were comparable rates of DVT in patients receiving ES alone or in combination with IPC. ${ }^{[10]}$ The use of IPC to prevent

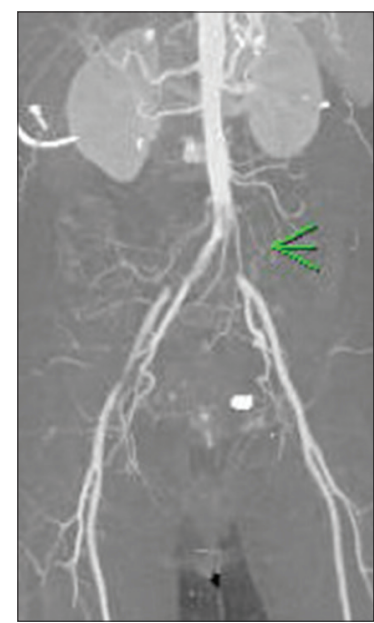

Figure 4: Computerised tomography angiogram demonstrating $L$ common iliac artery occlusion

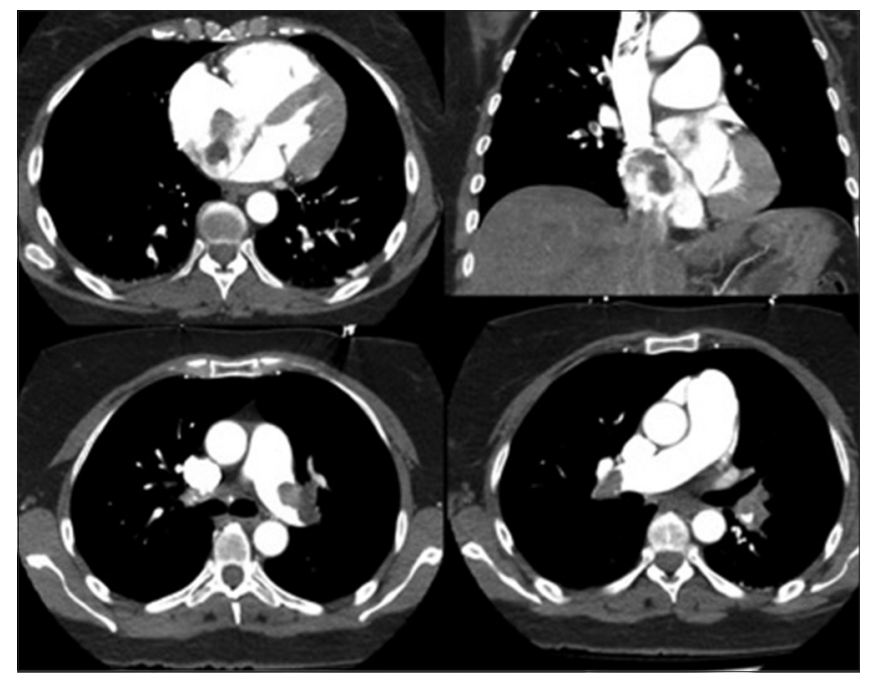

Figure 5: Computerised tomography pulmonary angiogram; bilateral pulmonary embolism, dilated right ventricular and intracardiac thrombus

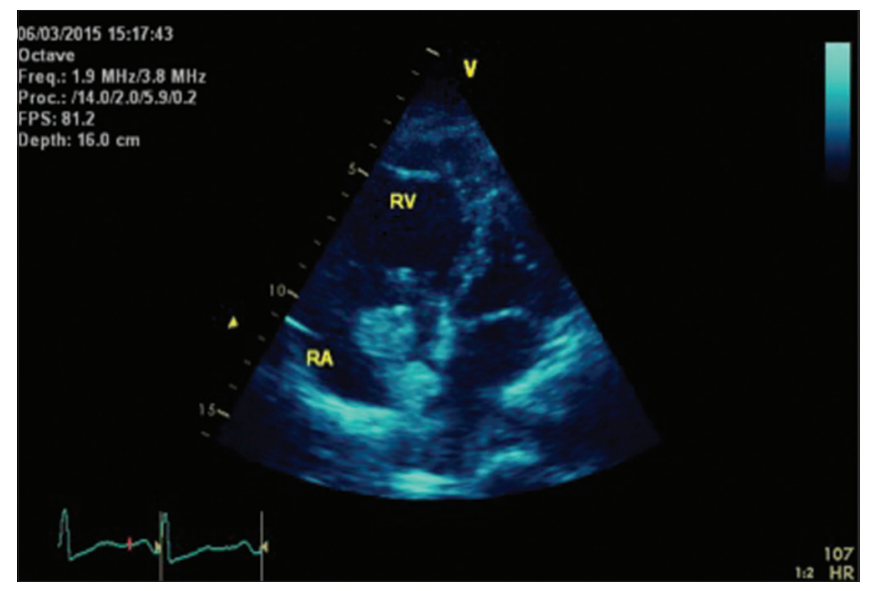

Figure 6: Transthoracic echocardiogram showing the right atrium thrombus crossing patent foramen ovale into the left atrium

VTE in neurosurgical patients is graded $2 \mathrm{C}$ evidence in the 2012 American College of Chest Physicians (ACCP) 
guidelines. ${ }^{[11]}$ The guidelines recommend IPC over $\mathrm{ES}$, although it concedes evidence for this, which is largely extrapolated from the CLOT-1 trial in stroke patients, where above-knee ES increased the rates of skin complications without significantly reducing the rates of DVT. ${ }^{[12]}$ Evidence that compares the efficacy of below- and above-knee devices is limited. However, a 2012 Cochrane review showed no difference between the two in general surgical populations. ${ }^{[13]}$ There is no clear guidance on optimal timing of commencing mechanical DVT prophylaxis, however a retrospective study of 207 patients found that the addition of IPC intraoperatively reduced DVT rates from $9.9 \%$ to $3.5 \% .{ }^{[14]}$ There is often a reluctance to continue IPC devices with established lower limb DVTs for the fear that this will increase the risk of embolic sequelae, however their application for an established DVT has been found to reduce the propagation of thrombus. ${ }^{[15]}$ Currently, there are no specific guidelines discussing this.

For pharmacological VTE prophylaxis in neurosurgical patients, the current options are usually LMWH and UFH. Other agents such as fondaparinux have not been studied in this patient group. A meta-analysis by Iorio and Agnelli of 1022 patients undergoing either craniotomy or spinal surgery suggested that the use of prophylactic heparins (UFH or LMWH) resulted in a $45 \%$ relative risk reduction for the development of VTE. ${ }^{[16]}$ A significant bleeding was observed in $2.3 \%$ of the patients who received UFH or LMWH and $1.4 \%$ of the patients who received placebo. Another meta-analysis conducted by Collen et al., ${ }^{[17]}$ including 7779 mixed neurosurgical patients from 30 studies, suggested the following: No VTE prophylaxis had the highest rates of DVT formation at $15.5 \%$, the use of either LMWH or UFH had significantly lower rates of DVT formation with no statistical difference between the two types of heparin when comparing the rates of intracranial haemorrhage $(\mathrm{ICH})$ and pooled relative risks did not approach a statistical significance but trended towards higher bleeding rates with heparin-based modalities, LMWH more so than UFH. Overall, there was no significant difference in the rates of ICH between heparin being administered pre-operatively, intraoperatively or postoperatively, although Dickinson et al. demonstrated high rates of ICH when LMWH prophylaxis was commenced pre-operatively, however this study was terminated early. ${ }^{[18]}$

Despite the ACCP guidelines recognising multiple risk factors for VTE, they dichotomise 'high' and 'very high' risks of VTE purely on the basis of the absence or presence of malignancy. They concluded that the benefit-risk ratio favoured IPC devices alone over pharmacologic prophylaxis in the 'high risk' (no malignancy) craniotomy group, whereas in the 'very high risk' (malignancy) group, they recommended adding pharmacological prophylaxis with either UFH or LMWH. This differentiation between high and very high risk on the basis of malignancy in these guidelines seems rather arbitrary. Perhaps, a potential individualised 'VTE-ICH' scoring tool similar to the 'CHADSVASC-HASBLED' for atrial fibrillation that incorporates all known risk factors for VTE as well as ICH, such as the presence of a vascular tumour or post-operative hypertension, would enable the recognition of the importance of tailored multimodal strategies. In addition to malignancy, our patient was subsequently found to be heterozygous for factor $\mathrm{V}$ Leiden. If known preoperatively, it may have prompted an extended duration of pharmacological prophylaxis beyond hospital stay, similar to the high-risk post-partum population, and also led to surveillance DVT scanning.

For established VTE complications applying conventional treatment strategies, post-craniotomy remains controversial due to the potential of life-threatening ICH, and data regarding the rates of peri-operative bleeding complications in neurosurgical patients are limited. Kalfas and Little reported a rate of $0.8 \%$ in 4992 patients undergoing intracranial procedures ${ }^{[19]}$ whereas Sheller et al. suggested an overestimation of the incidence of $\mathrm{ICH}$ associated with therapeutic anticoagulation in the post-craniotomy patients based on a retrospective study, in which none of the 42 patients developed $\mathrm{ICH}^{.20]}$ Meningioma surgery specifically requires extensive bone and soft tissue dissection, which may increase the risk of post-operative ICH associated with therapeutic anticoagulation; however, a study of 86 patients undergoing craniotomy for meningioma resection found no difference in the rates of ICH between those treated with or without LMWH. ${ }^{[21]}$

There is a poor evidence to suggest one method of systemic anticoagulation is superior to another for the treatment of established VTE in neurosurgical patients. Intravenous UFH has a shorter half-life and can be reversed more readily with protamine compared to LMWH. These factors are advantageous when considering the patients at risk of post-operative bleeding. However, they require titration as per the frequent APTT levels and can be difficult to keep within the desired therapeutic target, as demonstrated by our case. The mode of action of heparins is via antithrombin-mediated inhibition of serine proteases, and for UFH, this occurs predominantly via factor IIa (and Xa) inhibition, whereas for LMWH, this occurs mostly via factor Xa inhibition. Protamine neutralises anti-IIa activity and thus only partially reverses the LMWHs by $30-40 \%$. However, LMWHs do confer other advantages; no requirement for routine monitoring and dose adjustment except in renal impairment and greater bioavailability with longer 
duration of action when compared to UFH allowing once daily administration. There is also a reduced incidence of heparin-induced thrombocytopenia (HIT) and HIT with thrombosis syndrome.

\section{The role of inferior vena cava filters and thrombectomy}

There have been no studies looking at the use of IVC filters to prevent PE post-craniotomy, however there is conflicting evidence regarding their prophylactic use in patients post-spinal surgery. Rosner et al. ${ }^{[22]}$ recommended their use for PE prophylaxis in this high-risk group, whereas Davies et al. concluded that they have no role as a PE prophylaxis therapy. ${ }^{[23]} \mathrm{A}$ clinical trial of vena caval filters in the prevention of $\mathrm{PE}$ in patients with proximal DVT (PREPIC) showed that IVC filters reduced symptomatic PE rates from 15.1\% to $6.2 \%$; however, there was no mortality benefit at 8 years, and the rate of further symptomatic DVT was significantly higher in the filter group. ${ }^{[24]}$ A recent trial by Mismetti et al. compared the outcome of IVC filters in combination with anticoagulation versus anticoagulation alone on the incidence of recurrent PE. The trial showed no benefit from IVC filters in patients who are able to be anticoagulated. ${ }^{[25]}$ In addition, there are well-known complications associated with IVC filters such as device migration, embolisation of device components, perforation of the IVC, filter fracture and increased DVT formation at the insertion site.

The current guidance from the National Institute of Clinical Excellence is that IVC filters should be offered to patients with proven proximal DVT or $\mathrm{PE}$, only when anticoagulation is contraindicated or when alternative treatment options have failed. ${ }^{[26]}$ Our patient had an IVC filter placed with subsequent concomitant anticoagulation, as the risk of further VTE was deemed extremely high. Despite this intervention, she went on to have multiple recurrent PEs. There was no role for systemic thrombolysis due to the presence of a SDH, however other treatment options included catheter-directed thrombectomy (CDT) and surgical thrombectomy.

CDT can be either mechanical, rheolytic and/or intraclot thrombolytic injection. It does not result in the extraction of an intact thrombus, and fragments can be displaced distally. A meta-analysis of 35 studies supported the use of CDT for massive PE, ${ }^{[27]}$ and it is an ACCP low-level recommendation for emergency treatment of $\mathrm{PE}$ in patients where systemic thrombolytic therapy is contraindicated or has failed. ${ }^{[28]}$ Our patient required surgical excision of a right atrial thrombus, a paradoxical arterial embolus and repair of a PFO; therefore, surgical thrombectomy with cardiopulmonary bypass was the preferred option.

\section{Desmopressin for the treatment of diabetes} insipidus

Following craniotomy, patients can develop diabetes insipidus, which is commonly treated with desmopressin, a synthetic antidiuretic hormone. The main adverse effect of desmopressin is hyponatraemia, however desmopressin is also known to increase the plasma levels of von Willebrand factor (vWF) and factor VIII. It is used in the treatment of haemophilia A, von Willebrand's disease and patients with bleeding associated with uraemia due to end-stage renal disease. There are also reports of its utilisation to reduce blood loss in patients undergoing open-heart surgery. Furthermore, it is being investigated currently as a potential therapy in patients with intracerebral haemorrhage. ${ }^{[2]}$ High levels of vWF have been associated with arterial thrombosis and high levels of factor VIII with venous thrombosis. ${ }^{[30]}$

Desmopressin has more potent haematological effects than $\mathrm{ADH}$, and therefore the relatively small doses we use to treat DI may further elevate the risk of both venous and arterial thromboses in an already high-risk population. To our knowledge, there are no studies that have looked at desmopressin administration as an independent risk factor in this group. Until then, the balance of benefit versus harm remains unknown, and its use should be on a case-to-case basis.

\section{CONCLUSION}

The use of early multimodal prophylaxis combining mechanical and pharmacological methods appears to safely reduce the rates of VTE complications in patients post-craniotomy. Concerns regarding ICH are likely to persist, but we need to be cautious about overemphasising this risk due to the frequent development of VTE complications in this population. The high incidence of VTE in this cohort suggests we may not be getting the balance right. The heterogeneity of patients undergoing craniotomy means a 'one-size fits-all' approach may not work. The use of an individualised scoring system (similar to the 'CHADSVASC-HASBLED' for patients with atrial fibrillation) may facilitate an individualised approach to risk stratification. The concern for the development of ICH from therapeutic anticoagulation in post-craniotomy patients may be overstated, and an evidence-based suggestion to choose heparin modality is not suggested within the current literature.

While we need to clarify the role of our existing therapeutic armamentarium for the treatment of VTE complications, which currently includes heparin-based modalities and IVC filters, we should remain open to newer treatments such as CDT, which may prove to have greater benefit in this niche population. In certain 
contexts, traditional open thrombectomy may still have a role.

More importantly, it is imperative to maintain an open dialogue between the treating clinicians to ensure the avoidance of established dogma becoming further entrenched. In the modern climate, we are moving away from rigid evidence-based medicine to a more 'subjective' approach of 'individualised medicine'. However, individualised medicine is simply clinicians interpreting the evidence correctly and applying it in the correct contexts.

\section{Financial support and sponsorship Nil.}

\section{Conflicts of interest}

There are no conflicts of interest.

\section{REFERENCES}

1. Kimmell KT, Walter KA. Risk factors for venous thromboembolism in patients undergoing craniotomy for neoplastic disease. J Neurooncol 2014;120:567-73.

2. Smith TR, Nanney AD $3^{\text {rd }}$, Lall RR, Graham RB, McClendon J Jr., Lall RR, et al. Development of venous thromboembolism (VTE) in patients undergoing surgery for brain tumors: Results from a single center over a 10 year period. J Clin Neurosci 2015;22:519-25.

3. Hamilton MG, Hull RD, Pineo GF. Venous thromboembolism in neurosurgery and neurology patients: A review. Neurosurgery 1994;34:280-96.

4. Marras LC, Geerts WH, Perry JR. The risk of venous thromboembolism is increased throughout the course of malignant glioma: An evidence-based review. Cancer 2000;89:640-6.

5. Khaldi A, Helo N, Schneck MJ, Origitano TC. Venous thromboembolism: Deep venous thrombosis and pulmonary embolism in a neurosurgical population. J Neurosurg 2011;114:40-6

6. Agu O, Hamilton G, Baker D. Graduated compression stockings in the prevention of venous thromboembolism. Br J Surg 1999;86:992-1004.

7. Comerota AJ, Chouhan V, Harada RN, Sun L, Hosking J, Veermansunemi R, et al. The fibrinolytic effects of intermittent pneumatic compression: Mechanism of enhanced fibrinolysis. Ann Surg 1997;226:306-13.

8. Skillman JJ, Collins RE, Coe NP, Goldstein BS, Shapiro RM, Zervas NT, et al. Prevention of deep vein thrombosis in neurosurgical patients: A controlled, randomized trial of external pneumatic compression boots. Surgery 1978;83:354-8.

9. Morris RJ, Woodcock JP. Intermittent pneumatic compression or graduated compression stockings for deep vein thrombosis prophylaxis? A systematic review of direct clinical comparisons. Ann Surg 2010;251:393-6.

10. Turpie AG, Hirsh J, Gent M, Julian D, Johnson J. Prevention of deep vein thrombosis in potential neurosurgical patients. A randomized trial comparing graduated compression stockings alone or graduated compression stockings plus intermittent pneumatic compression with control. Arch Intern Med 1989;149:679-81.

11. Gould MK, Garcia DA, Wren SM, Karanicolas PJ, Arcelus JI, Heit JA, et al. Prevention of VTE in nonorthopedic surgical patients: Antithrombotic Therapy and Prevention of Thrombosis, $9^{\text {th }}$ ed: American College of Chest Physicians Evidence-based Clinical Practice Guidelines. Chest 2012;141 2 Suppl: e227S-77S.

12. CLOTS (Clots in Legs or sTockings after Stroke) Trial Collaboration. Thigh-length versus below-knee stockings for deep venous thrombosis prophylaxis after stroke: A randomized trial. Ann Intern Med 2010;153:553-62.

13. Sajid MS, Desai M, Morris RW, Hamilton G. Knee length versus thigh length graduated compression stockings for prevention of deep vein thrombosis in postoperative surgical patients. Cochrane Database Syst Rev 2012;5:CD007162.

14. Frisius J, Ebeling M, Karst M, Fahlbusch R, Schedel I, Gerganov V, et al. Prevention of venous thromboembolic complications with and without intermittent pneumatic compression in neurosurgical cranial procedures using intraoperative magnetic resonance imaging. A retrospective analysis. Clin Neurol Neurosurg 2015;133:46-54.

15. Partsch H. Ambulation and compression after deep vein thrombosis: Dispelling myths. Semin Vasc Surg 2005; $18: 148-52$.

16. Iorio A, Agnelli G. Low-molecular-weight and unfractionated heparin for prevention of venous thromboembolism in neurosurgery: A meta-analysis. Arch Intern Med 2000;160:2327-32

17. Collen JF, Jackson JL, Shorr AF, Moores LK. Prevention of venous thromboembolism in neurosurgery: A metaanalysis. Chest 2008;134:237-49.

18. Dickinson LD, Miller LD, Patel CP, Gupta SK. Enoxaparin increases the incidence of postoperative intracranial hemorrhage when initiated preoperatively for deep venous thrombosis prophylaxis in patients with brain tumors. Neurosurgery 1998;43:1074-81.

19. Kalfas IH, Little JR. Postoperative hemorrhage: A survey of 4992 intracranial procedures. Neurosurgery 1988;23:343-7.

20. Scheller C, Rachinger J, Strauss C, Alfieri A, Prell J, Koman G. Therapeutic anticoagulation after craniotomies: Is the risk for secondary hemorrhage overestimated? J Neurol Surg A Cent Eur Neurosurg 2014;75:2-6.

21. Cage TA, Lamborn KR, Ware ML, Frankfurt A, Chakalian L, Berger MS, et al. Adjuvant enoxaparin therapy may decrease the incidence of postoperative thrombotic events though does not increase the incidence of postoperative intracranial hemorrhage in patients with meningiomas. J Neurooncol 2009;93:151-6.

22. Rosner MK, Kuklo TR, Tawk R, et al. Prophylactic placement of an inferior vena cava filter in high-risk patients undergoing spinal reconstruction. Neurosurg Focus 2004;17(4):E6.

23. Davies MG, Hart JP, El-Sayed HF. Efficacy of prophylactic inferior vena caval filters in prevention of pulmonary embolism in the absence of deep venous thrombosis. J Vasc Surg Venous Lymphat Disord 2016;4:127-30.e1.

24. Decousus H, Leizorovicz A, Parent F, Page Y, Tardy B, Girard P, et al. A clinical trial of vena caval filters in the prevention of pulmonary embolism in patients with proximal deep-vein thrombosis. Prévention du Risque d'Embolie Pulmonaire par Interruption Cave Study Group. N Engl J Med 1998;338:409-15.

25. Mismetti P, Laporte S, Pellerin O, Ennezat PV, Couturaud F, Elias A, et al. Effect of a retrievable inferior vena cava filter plus anticoagulation vs. anticoagulation alone on risk of recurrent pulmonary embolism: A randomized clinical trial. JAMA 2015;313:1627-35.

26. NICE Guidelines [CG144]. Venous Thromboembolic Diseases: Diagnosis, Management and Thrombophilia Testing; 2012. Available from: http://www.nice.org.uk/guidance/CG144. [Last accessed on 2015 Nov 25].

27. Kuo WT, Gould MK, Louie JD, Rosenberg JK, Sze DY, 
Hofmann LV. Catheter-directed therapy for the treatment of massive pulmonary embolism: Systematic review and meta-analysis of modern techniques. J Vasc Interv Radiol 2009;20:1431-40.

28. Kearon C, Akl EA, Comerota AJ, Prandoni P, Bounameaux H, Goldhaber SZ, et al. Antithrombotic therapy for VTE disease: Antithrombotic Therapy and Prevention of Thrombosis, $9^{\text {th }} \mathrm{ed}$ : American College of Chest Physicians Evidence-Based Clinical
Practice Guidelines. Chest 2012;141 2 Suppl: e419S-94S.

29. Naidech AM, Maas MB, Levasseur-Franklin KE, Liotta EM, Guth JC, Berman M, et al. Desmopressin improves platelet activity in acute intracerebral hemorrhage. Stroke 2014;45:2451-3.

30. Kyrle PA, Minar E, Hirschl M, Bialonczyk C, Stain M, Schneider B, et al. High plasma levels of factor VIII and the risk of recurrent venous thromboembolism. N Engl J Med 2000;343:457-62. 